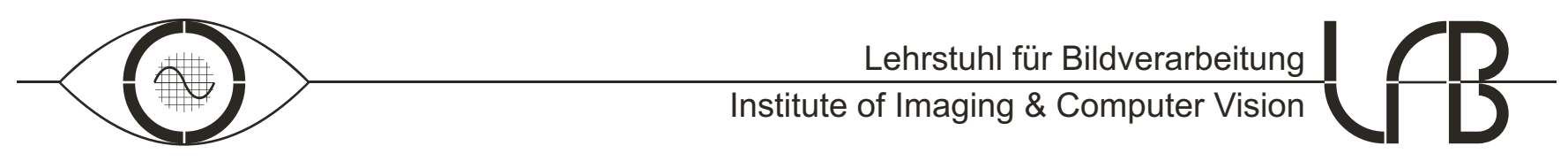

\title{
An Evaluation Framework for the Accuracy of Camera Transfer Functions Estimated from Differently Exposed Images
}

André A. Bell and Jens N. Kaftan and Dietrich Meyer-Ebrecht and Til Aach

Institute of Imaging and Computer Vision

RWTH Aachen University, 52056 Aachen, Germany

tel: +49 24180 27860, fax: +49241 8022200

web: www.lfb.rwth-aachen.de

in: 7th IEEE Southwest Symposium on Image Analysis and Interpretation. SSIAI 2006. See also $\mathrm{BIBT}_{\mathrm{E}} \mathrm{X}$ entry below.

BIBTEX:

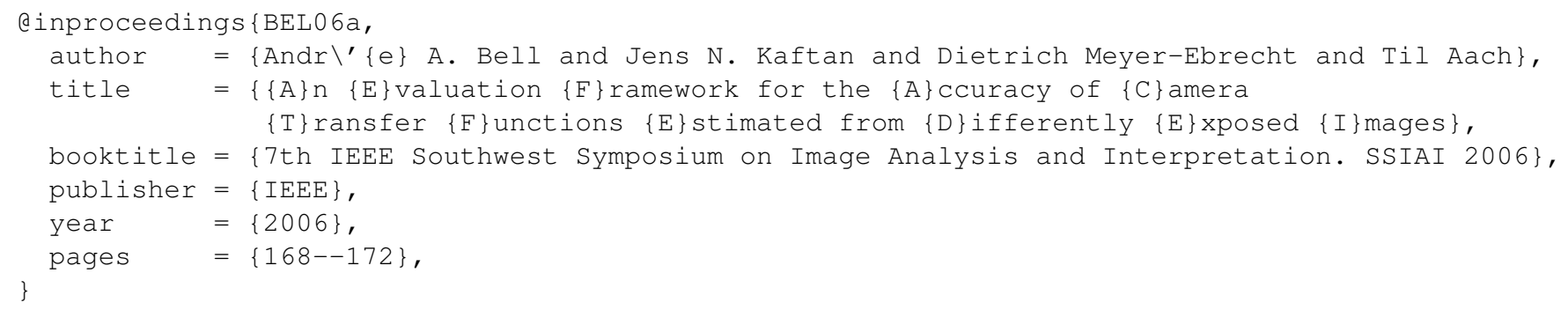

(C) 2006 IEEE. Personal use of this material is permitted. However, permission to reprint/republish this material for advertising or promotional purposes or for creating new collective works for resale or redistribution to servers or lists, or to reuse any copyrighted component of this work in other works must be obtained from the IEEE. 


\title{
An Evaluation Framework for the Accuracy of Camera Transfer Functions Estimated from Differently Exposed Images
}

\author{
André A Bell, Jens N Kaftan, Dietrich Meyer-Ebrecht, Til Aach \\ Institute of Imaging and Computer Vision, RWTH Aachen University, Germany \\ $\{$ bell,kaftan,meyer-ebrecht,aach $\} @ l f b . r w t h-a a c h e n . d e$
}

\begin{abstract}
Intensity values read from CCD- or CMOS-cameras are usually not proportional to the irradiance acquired by the sensor, but are mapped by a mostly nonlinear camera transfer function (CTF). This CTF can be measured using a test chart. This, however, is afflicted with the difficulty of ensuring uniform illumination of the chart. An alternative to chart-based measurements of the CTF is to use differently exposed images of the same scene. In this paper, we describe a radiometry-based experimental setup to directly measure the CTF. We furthermore show how to obtain image pairs of known exposure ratios from the same experiment, i.e., under identical environmental circumstances (light, temperature, camera settings). We use these images to estimate the CTF on differently exposed images, thus enabling a quantitative comparison of estimated and measured CTF.
\end{abstract}

\section{Estimating and Measuring the Camera Transfer Function}

Many algorithms in computer vision assume that the radiance of the scene is known. For instance, changes in scene radiance between images can be used to determine scene structure and illumination [12]. Also, the orientation of visible surfaces of the scene can be obtained from the radiance by shape from shading algorithms [13]. Radiance maps were moreover used to render synthetic objects more realistically into the scene [3].

In our application, we seek to tonally register cytopathological images taken at different exposures to generate a high dynamic range image. Image acquisition is based on a microscope equipped with a three-chip RGB color camera. Towards this end, it is crucial to determine the irradiance values in the image plane from the recorded intensity values. Unfortunately, the intensity values read from CCD- or CMOS-cameras are usually not proportional to the irradi- ance acquired by the sensor, but are mapped by the mostly nonlinear camera transfer function (CTF) $f$. In other words, we seek to apply the inverse $f^{-1}$ of the CTF $f$.

This CTF can be measured using a test chart like the Macbeth- or the CamAlign-CGH-chart, which consist of patches of known reflectance. Measuring the CTF using charts, however, is afflicted with the difficulty of ensuring uniform illumination of the chart. Furthermore it might not always be practicable since the CTF depends on parameter settings of the camera and the environment (e.g. temperature).

An alternative to chart-based measurements of the CTF is to use differently exposed images of the same scene $[10,8,9,4,11,5,6,1,2]$. Assuming that the exposure ratios of image pairs are known and the CTF can be modeled by, e.g., a $\gamma$-function

$$
I:=f(q)=\alpha+\beta q^{\gamma},
$$

the parameters $\alpha$ and $\gamma$ can be estimated by comparing the intensity values $f(q)$ of corresponding pixels in differently exposed images $[10,8,9]$. The scaling factor $\beta$ can not be recovered from these exposure sets and the parameter $q$, named the photoquantity in [8], denotes the amount of light received by a sensor element. Such a parametric model can also be replaced by a smoothness constraint [4]. Alternatively a polynomial [11] or a constrained piecewise linear model has been used [1,2]. It has been shown that one needs to either know the exposure ratios or make an assumption about the CTF [5, 6].

These methods offer appealing ways to recover the CTF, even in a non-laboratory environment. So far the accuracy of the estimated CTFs has been evaluated qualitatively with CamAlign-CGH measurements plotted into the curve of the recovered CTF [9] or using a Macbeth chart instead [5]. The influence of noisy measurements has been shown to be less than $2.7 \%$ in synthetic data [11].

In this paper, we describe a radiometry-based experimental setup to directly measure the CTF. We furthermore show how to obtain image pairs of known exposure ratios from 
the same experiment, viz. under identical environmental circumstances (light, temperature, camera settings). We use these images to estimate the CTF by one of the above methods, thus enabling a quantitative comparison of estimated and measured CTF.

\section{Experimental Setup}

Our CTF measurements are based essentially on a homogeneous light source realized by an integrating sphere (U1brichtsphere). An integrating sphere provides an isotropic and homogeneous light output in terms of radiance $L$ (measured in $\left.\frac{W}{s r \cdot m^{2}}\right)$ at its opening of diameter $r$ [7]. We illuminate the camera sensor directly by this light source, with all optics removed to ensure homogeneous illumination over the entire sensor area, and to avoid distortions introduced by the optics. The irradiance impinging on the sensor is then given by [7]

$$
E=\pi \frac{r^{2}}{r^{2}+x^{2}} L
$$

where $x$ is the distance between sensor and exit pupil of the light source. The image acquisition process integrates this irradiance $E$ (measured in $\frac{W}{m^{2}}$ ) over exposure time $t$ and area $A$ of a sensor element, yielding the energy

$$
Q=A E t
$$

detected by that sensorelement. $Q$ corresponds to

$$
N_{p}=A E t \frac{\lambda}{h c}
$$

detected photons, where $h$ is Planck's constant and $c$ is the speed of light. $N_{p}$ maps to the sensor signal $q$ via the quantum efficiency $\eta$ of a sensor element according to

$$
q=\eta A E t \frac{\lambda}{h c}
$$

In non-monochromatic light, $\eta$ and $E$ may depend on the wavelength $\lambda$, the total response $q$ is then given by integrating (3) over $\lambda$ [7]. Additionally, in case of a color camera, the irradiance incident on the sensor will be filtered by colorfilters (e.g., Bayer color-filter-array) beforehand. The quantity of light $q$ undergoes further mostly nonlinear transformations described by the $\mathrm{CTF} f$ such as, e.g., dynamic range compression and quantization.

To measure the CTF in our experimental setup, we record the camera response $f(q)$ for different known distances $x$. Samples of the CTF are then straightfowardly obtained from (2) and (3).

Additionally, for every image taken at a distance $x_{1}$, we acquire a second image at position

$$
x_{2}=\sqrt{r^{2}+2 x_{1}^{2}}
$$

which, according to (2), receives half of the irradiance in the image plane. The image pairs of exposure ratio $k=2$ recorded this way are used to estimate the CTF like in $[10$, $8]$.

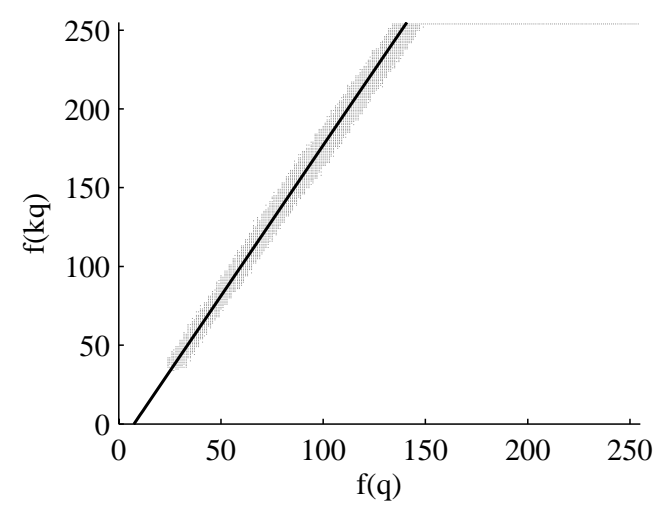

\section{Figure 1. Joint histogram example of the JAI CV-M90 red channel.}

\section{Estimating the Camera Transfer Function}

Let us consider two images $f_{1}$ and $f_{2}$ with exposure ratio $k=2$, such that $f_{2}$ receives half of the irradiance as $f_{1}$. This is equivalent to observing the quantity of light $q$ and $k q$ through the same CTF $f$. Based on such an image pair the joint histogram between these two images $[10,8]$ can be computed. In case of having more image pairs of equal exposure ratio $k$ these can be added to the joint histogram. Afterwards the function $g(f)$, defined by

$$
g(f(q))=f(k q)
$$

has to be estimated from this joint histogram. Choosing the camera model (1) gives

$$
\begin{aligned}
g(f) & =f(k q) \\
& =\alpha+\beta(k q)^{\gamma} \\
& =\alpha-k^{\gamma} \alpha+k^{\gamma} \alpha+k^{\gamma} \beta q^{\gamma} \\
& =\alpha\left(1-k^{\gamma}\right)+k^{\gamma}\left(\alpha+\beta q^{\gamma}\right) \\
& =\alpha\left(1-k^{\gamma}\right)+k^{\gamma} f
\end{aligned}
$$

which is a straight line in the joint histogram between the toe- and shoulder-region [8]. The slope $m$ and intercept $b$ are therefore given by $m=k^{\gamma}$ and $b=\alpha\left(1-k^{\gamma}\right)$. After fitting a line into the joint histogram, it is possible to determine the parameter $\alpha$ and $\gamma$ of the camera model by 


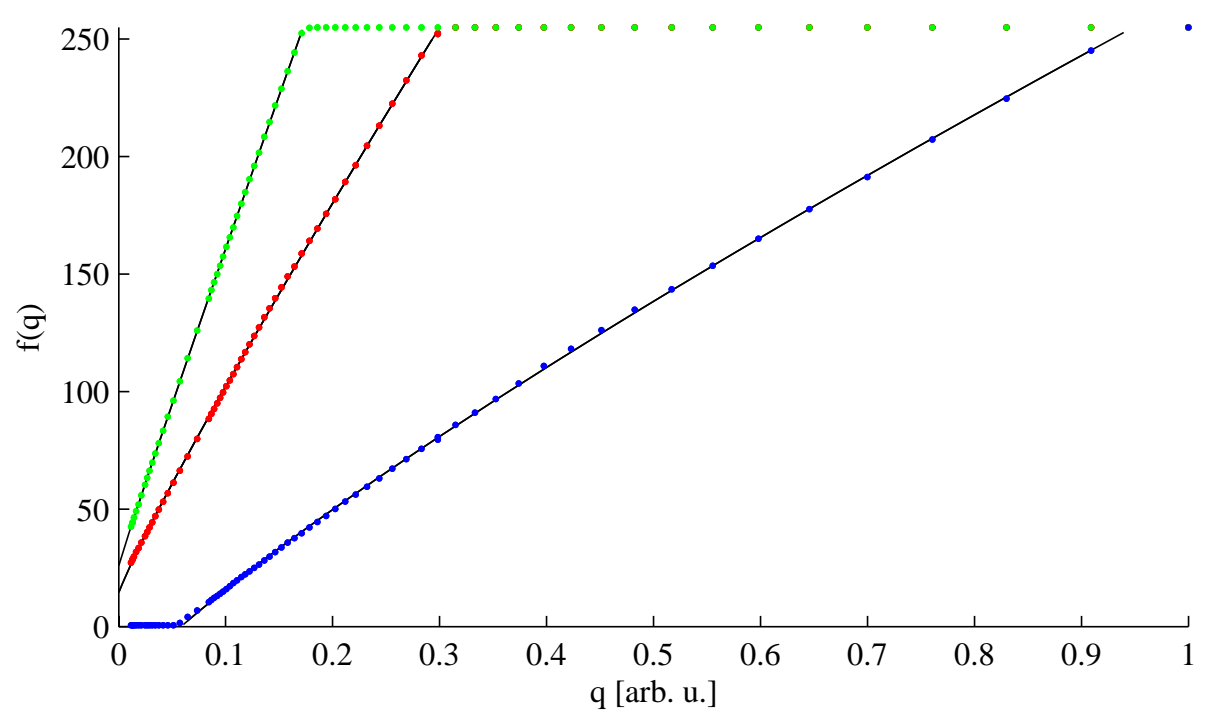

Figure 2. Measured datapoints and fitted CTF for a JAI CV-M90 3-chip-CCD (8bit) camera.

$$
\begin{aligned}
\gamma & =\frac{\log m}{\log k} \\
\alpha & =\frac{b}{1-m}
\end{aligned}
$$

Figure 1 shows a plot of a joint histogram generated from 60 image pairs of exposure ratio $k=2$ obtained as described in section 2. The line has been fitted into the datapoints between the toe and shoulder region using a linear regression $\chi^{2}$-fitting. From the estimated values $m=1.9109$ and $b=-14.207$ and by using (5) and (6) one obtains $\gamma=0.9343$ and $\alpha=15.5967$ as parameter for the camera model.

\section{Results}

Based on (1) as a model for the 3-chip RGB camera JAI CV-M90, we estimated the parameters $\alpha$ and $\gamma$. Range limits are not accounted for in this model. Therefore $\alpha$ corresponds to the dark current for positive values only. For negative $\alpha$, the CTF is truncated to zero. Towards higher values the $\mathrm{CTF}$ exhibits the expected saturation due to the limited maximum ("full-well") charge generation capacity of the sensor. The dynamic compression of the camera is captured by the parameter $\gamma$. For the measurements, the parameter $\beta$ is determined by the radiance $L$ of the light source. Since $L$ cannot be recovered by estimation from sets of differently exposed images, we performed a leastsquares fit of the estimated CTF to the measured CTF with respect to this parameter for comparison. Results are shown in figure 2. Since $\gamma \neq 1$ the CTFs are not linear functions. Evidently, as can be seen from figure 2, the fitted CTF represents the measured data of a JAI CV-M90 3-chip-CCD camera very precisely. The mean absolute difference between these data points and the fitted model of the measured CTF is $\mu_{\text {red }}=0.4973, \mu_{\text {green }}=0.1870$, and $\mu_{\text {blue }}=0.4705$ intensity values for the red, green, and blue channel respectively, which proves that the model assumption is valid for this camera. Table 1 shows the measured and estimated parameters for each color channel of the JAI CV-M90 3-chipCCD camera. The mean absolute error between measured and estimated CTF is $\mu_{\text {red }}=0.4641, \mu_{\text {green }}=0.4969$, and $\mu_{\text {blue }}=0.9856$ for red, green, and blue channel respectively.

Table 1. Comparison of measured and estimated parameters $\alpha$ and $\gamma$ for each color channel of the JAI CV-M90 3-chip-CCD camera.

\begin{tabular}{|c|c|c|}
\hline & Measured CTF & Estimated CTF \\
\hline$\alpha_{\text {red }}$ & 14.5919 & 15.5967 \\
\hline$\gamma_{\text {red }}$ & 0.9210 & 0.9343 \\
\hline$\alpha_{\text {green }}$ & 25.9173 & 24.6797 \\
\hline$\gamma_{\text {green }}$ & 0.9723 & 0.9706 \\
\hline$\alpha_{\text {blue }}$ & -27.9872 & -23.3515 \\
\hline$\gamma_{\text {blue }}$ & 0.8300 & 0.8817 \\
\hline
\end{tabular}




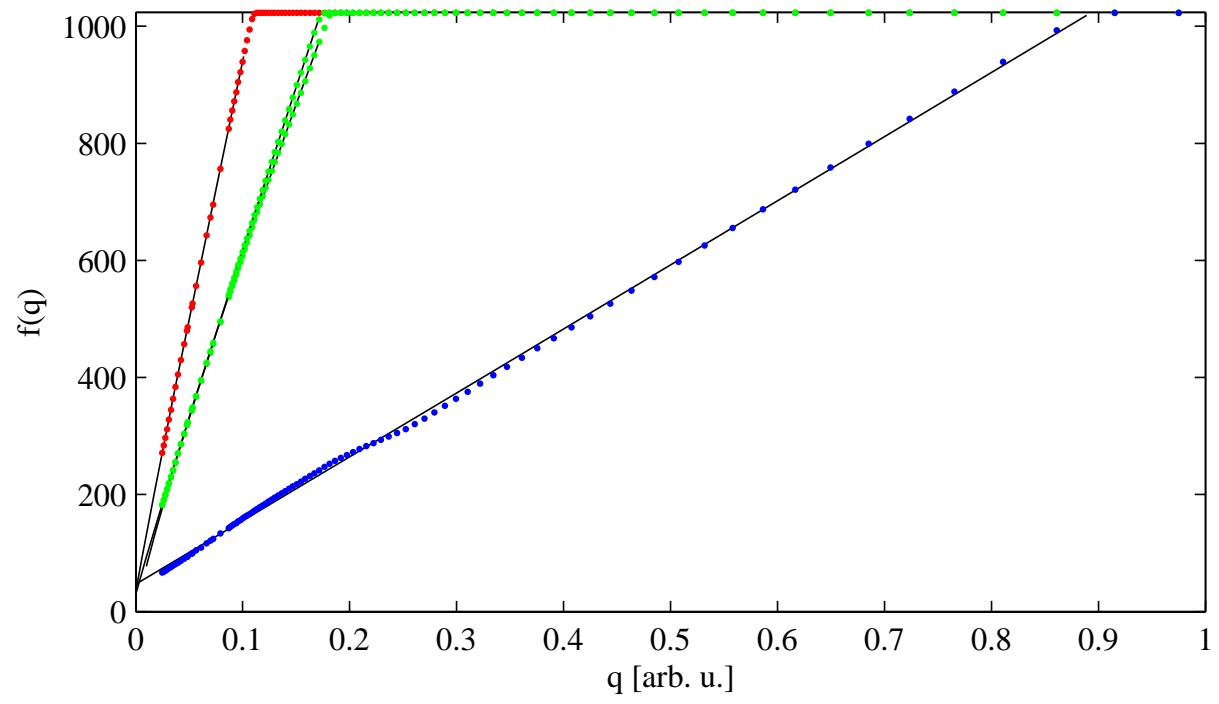

Figure 3. Measured datapoints and fitted CTF for a AVT Dolphin F145C single chip Bayer color-filterarray (10bit) camera (b).

Alternatively we have chosen the same camera model (1) for the AVT Dolphin F145C single chip Bayer colorfilter-array camera. We estimated $\alpha$ and $\gamma$ and measured the CTF accordingly. As can be seen in figure 3, the camera model (1) does not fit as good as for the 3-chip camera. The displayed curves in figure 3 represent the color channels corresponding to the rasters of the Bayer pattern. Therefore we have two green channels. The mean absolute difference between the measured data points and the fitted model of the CTF is $\mu_{\text {red }}=0.6591, \mu_{\text {green } 1}=1.5541$, $\mu_{\text {green } 2}=0.6728$, and $\mu_{\text {blue }}=5.4489$ intensity values for the red, green1, green2, and blue channel respectively. It should be noticed that the two green channels diverge as soon as red is going to be saturated and that blue is to be dented as soon as the second green channel reaches the saturation point (see figure 3). This effect may be due to blooming from one color channel into the other, which is an effect that can not occur for a 3-chip camera. Table 2 shows the measured and estimated parameters for each color channel of the AVT Dolphin F145C single chip Bayer colorfilter-array camera. For comparison we have carried out a least-squares fit of the estimated CTF to the measured CTF with respect to the parameter $\beta$ as in the case of the other camera. The mean absolute error between measured and estimated CTF is $\mu_{\text {red }}=0.6555, \mu_{\text {green } 1}=1.8828$, $\mu_{\text {green } 2}=0.7635$, and $\mu_{\text {blue }}=6.509$ intensity values in mean for red, green1, green 2 and blue respectively.
Table 2. Comparison of measured and estimated parameters $\alpha$ and $\gamma$ for each color channel of the AVT Dolphin F145C single chip Bayer color-filter-array camera.

\begin{tabular}{|c|c|c|}
\hline & Measured CTF & Estimated CTF \\
\hline$\alpha_{\text {red }}$ & 34.6273 & 34.1294 \\
\hline$\gamma_{\text {red }}$ & 0.9654 & 0.9648 \\
\hline$\alpha_{\text {green } 1}$ & -7.9983 & 0.7144 \\
\hline$\gamma_{\text {green } 1}$ & 0.8618 & 0.8822 \\
\hline$\alpha_{\text {green } 2}$ & 29.5660 & 29.8274 \\
\hline$\gamma_{\text {green } 2}$ & 0.9628 & 0.9615 \\
\hline$\alpha_{\text {blue }}$ & 46.6789 & 27.7228 \\
\hline$\gamma_{\text {blue }}$ & 1.0037 & 0.9130 \\
\hline
\end{tabular}

\section{Discussion}

We have shown that it is possible to directly measure the CTF and, under the same environmental circumstances, to acquire image pairs with known exposure ratio. The directly measured CTF can be used to verify the accuracy of the assumed camera model or to find a camera model for a specific camera. From the simultaneously acquired image pairs of known exposure ratio one can estimate the CTF using the same algorithms which are available for nonlaboratory conditions. Both measured and estimated CTF, 
can now be quantitatively compared, i.e., this experiment will either falsify the camera model or demonstrate the quality of estimated CTF under normal circumstances. Additionally this method can give insight into the limitations of an estimation algorithm for the CTF, which can be used to improve the algorithm. We intend to evaluate further estimation algorithms and a larger variety of cameras, in the near future.

\section{References}

[1] F. M. Candocia. Analysis and enhancements to piecewise linear comparametric image registration. IEEE Transactions on Image Processing, 14(2):181-188, 2005.

[2] F. M. Candocia and D. A. Mandarino. A semiparametric model for accurate camera response function modeling and exposure estimation from comparametric data. IEEE Transactions on Image Processing, 14(8):1138-1150, 2005.

[3] P. E. Debevec. Rendering synthetic objects into real scenes. In Proceedings ACM SIGGRAPH, pages 189-198, 1998.

[4] P. E. Debevec and J. Malik. Recovering High Dynamic Range Radiance Maps from Photographs. In Proceedings ACM SIGGRAPH, pages 369-378, 1997.

[5] M. D. Grossberg and S. K. Nayar. What can be Known about the Radiometric Response from Images? In European Conference on Computer Vision. ECCV 2002, volume 4, pages 189-205, 2002.

[6] M. D. Grossberg and S. K. Nayar. Determining the Camera Response from Images: What Is Knowable? IEEE Transactions on Pattern Analysis and Machine Intelligence, 25(11):1455-1467, 2003.

[7] B. Jähne. Practical handbook on image processing for scientific and technical applications. CRC Press LLC, 2nd edition, 2004.

[8] S. Mann. Comparametric equations with practical applications in quantigraphic image processing. IEEE Transactions on Image Processing, 9(8):1389-1406, 2000.

[9] S. Mann and R. Mann. Quantigraphic Imaging: Estimating the camera response and exposures from differently exposed images. In IEEE Conference on Computer Vision and Pattern Recognition. CVPR 2001, volume 1, pages 842-849, 2001.

[10] S. Mann and R. W. Picard. Being 'undigital' with digital cameras: Extending Dynamic Range by Combining Differently Exposed Pictures. Technical Report 323, M.I.T. Media Lab Perceptual Computing Section, Boston, Massachusetts, 1994.

[11] T. Mitsunaga and S. K. Nayar. Radiometric self calibration. In IEEE Conference on Computer Vision and Pattern Recognition. CVPR 1999, volume 1, pages 374-380, 1999.

[12] S. K. Nayar, K. Ikeuchi, and T. Kanade. Shape from Interreflections. In International Conference on Computer Vision. ICCV 1990, pages 2-11, 1990.

[13] R. Zhang, P.-S. Tsai, J. E. Cryer, and S. Mubarak. Shape from Shading: A Survey. IEEE Transactions on Pattern Analysis and Machine Intelligence, 21(8):690-706, 1999. 